\title{
Return to system within 30 days of discharge following pediatric shunt surgery
}

\author{
Clinical article
}

\author{
Joshua J. Chern, M.D., Ph.D., ${ }^{1,2}$ Markus Bookland, M.D., ${ }^{1}$ Javier Tejedor-Sojo, M.D. . ${ }^{3}$ \\ Jonathan Riley, M.D., ${ }^{2}$ Mohammadali M. Shoja, M.D., ${ }^{4}$ R. Shane Tubbs, Ph.D., P.A.-C., ${ }^{4}$ \\ AND ANDREW REISNER, M.D. ${ }^{1,2}$
}

${ }^{1}$ Pediatric Neurosurgery Associates and ${ }^{3}$ Department of Pediatrics, Children's Healthcare of Atlanta;

${ }^{2}$ Department of Neurosurgery, Emory University, Atlanta, Georgia; and ${ }^{4}$ Division of Neurosurgery,

Department of Surgery, University of Alabama at Birmingham, Alabama

\begin{abstract}
Object. The rate of readmission after CSF shunt surgery is significant and has caught the attention of purchasers of health care. However, a detailed description of clinical scenarios that lead to readmissions and reoperations after index shunt surgery is lacking in the medical literature.

Methods. This study included 1755 shunt revision and insertion surgeries that were performed at a single institution between May 1, 2009, and April 30, 2013. Demographic, socioeconomic, and clinical characteristics were prospectively collected in the administrative, business, and operating room databases. Clinical events within the 30 days following discharge were reviewed and analyzed. Two events of interest, Emergency Department (ED) utilization and reoperation, were further analyzed for risk factor associations by using multivariate logistic regression.

Results. There were 290 readmissions within 30 days of discharge (16.5\%). Admission sources included ED (n $=216)$, hospital transfers $(\mathrm{n}=23)$, and others. Of the 290 readmissions, 184 were associated with an operation, but only 165 of these were performed by the neurosurgical service. These included surgeries for shunt occlusion and externalization $(n=150)$, wound revision $(n=7)$, and other neurosurgical procedures that were not shunt related $(n=$ 8). The remaining readmissions $(n=106)$ were not associated with an operation, and only 59 patients were admitted for issues related to the index shunt surgery.

When return to the ED was the dependent variable in a multivariate regression model, patients who returned to the ED were more likely to be from the Atlanta metropolitan area and to be either uninsured or insured with public assistance. When reoperation was the dependent variable, patients whose surgery started after 3 p.m. were more likely to undergo subsequent CSF shunt revision surgery on readmission.

Conclusions. Of the readmissions within 30 days of shunt surgery, $74.5 \%$ were related to the index shunt surgery. Whether and to what extent these readmissions are preventable continues to be controversial. Further study is needed to identify modifiable risk factors that may eventually improve patient care.

(http://thejns.org/doi/abs/10.3171/2014.2.PEDS13493)
\end{abstract}

$\begin{array}{lccc}\text { KEY WORDS } & \text { hospital readmission } & \bullet \quad \text { cerebrospinal fluid shunt } \\ \text { quality control } & \bullet \quad \text { administrative claims data } \bullet \quad \text { hydrocephalus }\end{array}$

$\mathrm{R}$

EADMISSION after discharge is widely used as a quality measure, even though its clinical significance is still controversial. $5,8,10$ The ideal goal is to identify preventable readmissions, which when eliminated would presumably reflect improvement in patient care. In addition, it would also imply a cost saving that is highly sought after in today's health care environment.

Multiple studies have identified CSF shunt surgeries as a potential area of improvement because of the

\footnotetext{
Abbreviations used in this paper: $\mathrm{CPT}=$ current procedural terminology; ED = Emergency Department; LOS = length of hospital stay; NACHRI = National Association of Children's Hospitals and Related Institutions; NSQIP = National Surgical Quality Improvement Program.
}

relatively high readmission and reoperation rate..$^{1,3}$ Consequently, this has also been cited as the surgical category with the greatest readmission cost. From a neurosurgeon's standpoint, there are 3 general areas of concern. First, analytical results derived from an administrative database need to be verified. Second, despite the emphasis on shunt reoperations, to what degree is a shunt malfunction preventable? From numerous studies conducted over decades, there is a basal rate of shunt malfunction that is commonly acknowledged by neurosurgeons but less so by other medical professionals. ${ }^{7}$ Third, whereas reoperation is highly visible and relatively easy to identify, there are various other clinical events that occur after shunt surgery. A description of each of these nonsurgical events is not currently available. If one would argue that shunt 


\section{J. J. Chern et al.}

malfunction is largely unavoidable, there might be other preventable events that are overlooked.

In this study we first describe various clinical events that occurred after a shunt insertion or revision in a single institution. We then analyze clinical and demographic factors associated with different clinical end points. A brief discussion on preventability then follows.

\section{Methods}

\section{Study Population and Data Fields Used}

This study included $1755 \mathrm{CSF}$ shunt revision and insertion surgeries that were performed at a single institution between May 1, 2009, and April 30, 2013, by boardcertified and board-eligible pediatric neurosurgeons and fellows. Demographic, socioeconomic, and clinical characteristics were prospectively collected in the administrative, business, and operating room databases, including EPIC ASAP (emergency department application), OpTime (surgical application), EpicCare Inpatient (universal hospital system), EpicCare Ambulatory (outpatient medical record application), and ADT (inpatient and outpatient admission-discharge-transfer application). Data from the individual database were then merged into a single operational database on an Oracle platform (Oracle 11g). Sequential hospital system visits within 30 days of discharge were then queried and imported into a Microsoft Excel file for further analysis.

Demographic data fields that were collected at the time of index shunt surgery included age, primary language, race/ethnicity, zip code, and primary payer status (public assistance, self pay vs private insurance). Two data fields were derived from the zip code in conjunction with US population census data from 2010: 1) average income; and 2) whether the zip code belonged to the Atlanta metropolitan area.

Data fields that were related to the index shunt surgeries and all surgeries during readmissions included surgeon identity, length of hospital stay (LOS), procedure length, starting time of the procedure, admission source (elective admission vs admission from the Emergency Department [ED]), current procedural terminology (CPT) code and surgeon-dictated procedure comments (a short description entered by operating room circulating nurses), and operative reports. If surgery began after 3 p.m. but before 7 a.m., it was labeled as an "after-hour" surgery. Admissions to pediatric and neonatal ICUs were also captured.

Distinctions were made among ventriculoatrial shunts, subdural/cyst shunts, lumboperitoneal shunts, and shunt procedures that were preceded by shunt externalization or external ventricular drain insertions. Hospital stays that were $\geq 3$ days and patients with myelomeningocele were categorized. A categorical variable was defined for surgeries in which length was $>1$ SD above the average.

\section{Statistical Methods}

Statistical analysis was performed with IBM SPSS Statistics version 20. The associations between dichotomous variables were analyzed with the Fisher exact test. Binary logistic regression analysis was used to assess whether significant risk factors identified from univariate analysis independently contributed to the increased hazard of dependent variables. The LOS (in days) and procedure length (in minutes) were analyzed as continuous variables in the regression model but also presented as a categorical variable for clarity. Tolerance statistics $<0.20$ were considered to reflect colinearity. A 2-tailed $\mathrm{p}$ value $<0.05$ was considered statistically significant.

\section{Results}

\section{Determination of Study Cohort and Patient Characteristics}

Shunt surgeries were defined based on CPT codes (62190 to 62258, excluding 62200 and 62201). Additional procedures and details of the procedure were identified from surgeon-dictated procedure comments and operative reports. There were 1850 shunt surgeries in the 4-year period. Patients who were discharged to other hospital systems were excluded. For example, neonates who underwent shunt insertion followed by transfer to the original hospital were excluded. A total of 95 surgeries were excluded; this left 1755 in the study cohort. Descriptive statistics of the patient cohort and the surgeries are provided in Table 1.

\section{Sources of Readmissions Within 30 Days of Discharge}

A summary of readmission sources and events occurring after the readmission is shown in Fig. 1. The "allcause" readmission rate within 30 days of index ventricular shunt surgery was $16.5 \%$ (290 of 1755). Within 30 days of discharge, there were $375 \mathrm{ED}$ visits that resulted in 216 readmissions. A considerable number of patients were discharged home from the ED $(n=159,42 \%)$. Twenty-three readmissions were transfers from other hospitals. The most common scenario was a patient who lived far away who went to the local ED for evaluation. He or she was then transferred to our hospital for further care if shunt malfunction was suspected. There were 5 planned readmissions for neurosurgical procedures (suture removal, cranial encephalocele closure, Chiari decompression, vagal nerve stimulator placement, and cranioplasty). There were 35 planned and unplanned readmissions by other (nonneurosurgical) services for both surgical ( $\mathrm{n}=$ 19) and nonsurgical procedures (for example, video EEG).

There were 1482 postoperative neurosurgery clinic visits, but only 1158 occurred within 30 days after discharge. Seventy-eight patients did not have any clinic visits after surgery. There were 11 direct admissions from the neurosurgical outpatient clinic when a problem was identified.

\section{Events After Readmission}

For 184 readmissions there was an association with one or more operations during the hospitalization (Fig. 1). Shunt revision, shunt infection, and wound revision accounted for 157 of the 184 operations. Therefore, the overall shunt revision rate within the first 30 days was $8.9 \%$ (157 of 1755). In the 106 admissions that were not associated with an operation, Neurosurgery was the primary admission and/or discharge service in 59 encoun- 


\section{Return to system after shunt surgery}

TABLE 1: Characteristics and other variables associated with index shunt surgery in 1755 patients with hydrocephalus*

\begin{tabular}{|c|c|c|}
\hline Variable & Value $(\%) \dagger$ & Range \\
\hline \multicolumn{3}{|l|}{ continuous } \\
\hline age in yrs & $7.15 \pm 6.67$ & $0-24$ \\
\hline LOS in days & $6.67 \pm 17.2$ & $1-268$ \\
\hline op length in mins & $42.0 \pm 27.4$ & $11-252$ \\
\hline median HI per zip code, in US\$ & 47,514 & $13,084-114,674$ \\
\hline \multicolumn{3}{|l|}{ categorical } \\
\hline admission from ED before index shunt op & $961(54.8)$ & \\
\hline ICU admission after shunt revision & $354(20.2)$ & \\
\hline NICU admission & $152(8.7)$ & \\
\hline English as primary language & $1619(92.2)$ & \\
\hline Caucasian & $883(50.3)$ & \\
\hline Atlanta metropolitan zip codes & $661(37.7)$ & \\
\hline op started after 3 p.m. \& before 7 a.m. & $512(29.2)$ & \\
\hline Medicaid/Medicare as the primary payee & $1010(57.5)$ & \\
\hline insertion/revision of VAS & $112(6.4)$ & \\
\hline insertion/revision of subdural/cyst shunt & $136(7.7)$ & \\
\hline insertion/revision of LPS & $52(3.0)$ & \\
\hline externalization of shunt/EVD removal, placement & $104(5.9)$ & \\
\hline LOS $\geq 3$ days & $702(40)$ & \\
\hline op length $>70$ mins & $189(10.8)$ & \\
\hline patients w/ spina bifida & $140(8.0)$ & \\
\hline \multicolumn{3}{|l|}{ provider } \\
\hline 1 & $549(31.3)$ & \\
\hline 2 & $351(20)$ & \\
\hline 3 & $392(22.3)$ & \\
\hline 4 & $277(15.8)$ & \\
\hline 5 & $96(5.5)$ & \\
\hline 6 & $30(1.7)$ & \\
\hline 7 & $60(3.4)$ & \\
\hline
\end{tabular}

* EVD = external ventricular drain; $\mathrm{HI}=$ household income; LPS = lumboperitoneal shunt; NICU = neonatal ICU; VAS = ventriculoatrial shunt.

$\dagger$ The first 3 entries in the column are expressed as the mean \pm SD. The values for categorical variables represent the number of patients (\%).

ters. Headache, nausea, and vomiting were the most common admission complaints. Thus, admissions related to index shunt surgery accounted for $74.5 \%$ of readmissions (216 of 290).

In the category of nonneurosurgery admissions ( $\mathrm{n}$ $=66$ ), 47 were nonsurgical. The most common admission services for nonsurgical patients were Hematology/ Oncology $(n=14)$, Pediatrics $(n=12)$, Neurology $(n=8)$, and Gastroenterology $(n=5)$.

\section{Risk Factors Associated With Patient Subgroups}

Multivariate analysis was used to identify clinical and demographic factors that were predictive of clinical events following shunt surgeries. Two clinical events were analyzed as dependent variables: 1) return to ED; and 2) shunt reoperation. Both of these clinical events overlap to a certain extent (some of the patients who returned to the operating room would have been readmitted from the ED), but the former would have included patients who were discharged from the ED as well as patients who were admitted but did not undergo any surgical procedures. Results from univariate and multivariate analyses are presented in Tables 2-4.

Significant risk factors identified from univariate analysis (Table 2) were used to construct multivariate analysis models. Surgeon identity was tested using contingency tables and did not significantly correlate with shunt revision or readmission rates. Using return to ED as a dependent variable $(n=375)$, multivariate logistic regression identified Atlanta zip codes and Medicaid payee 


\section{J. J. Chern et al.}

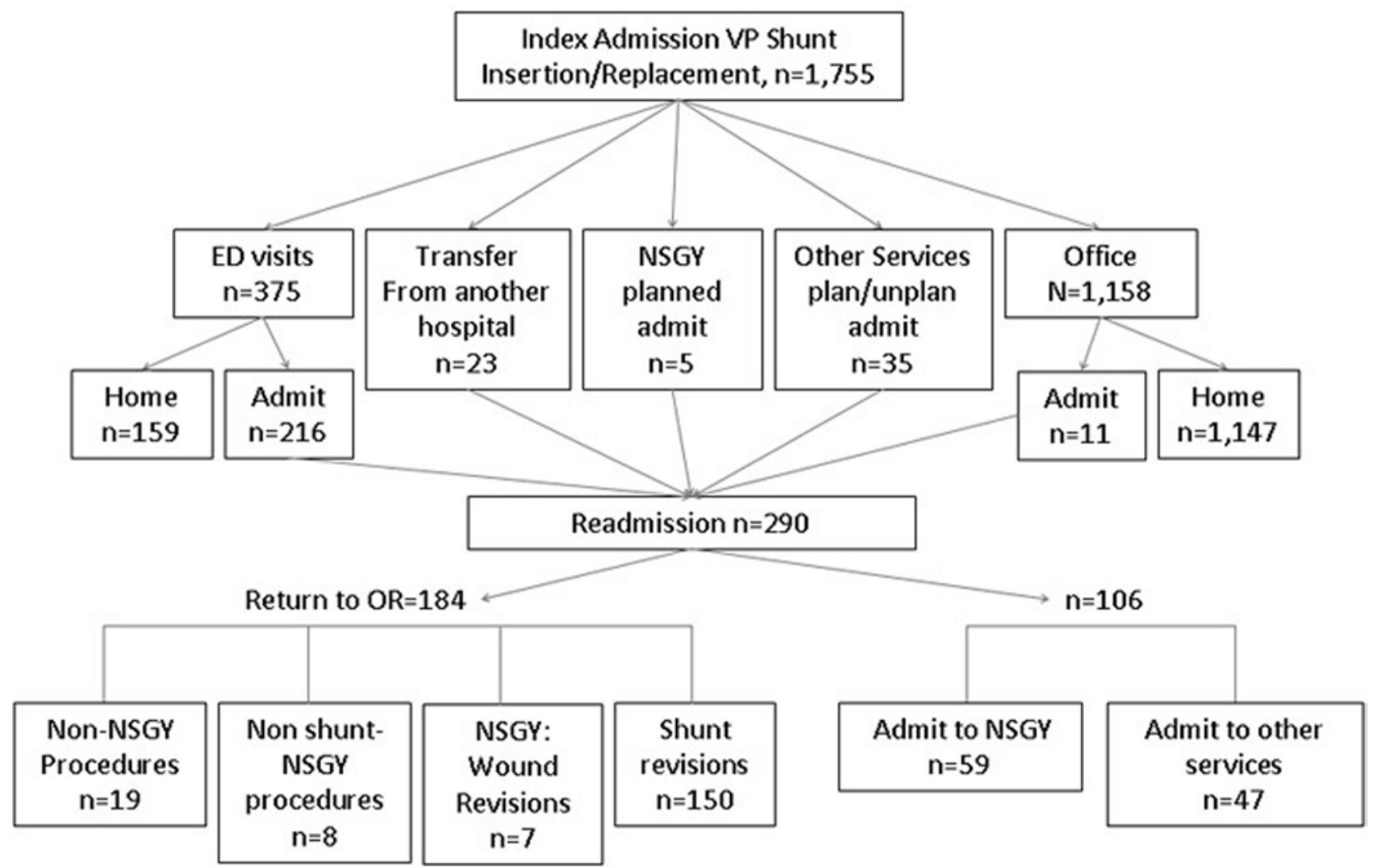

FIG. 1. Flow chart showing 30 -day clinical events after 1755 shunt surgeries over a 4 -year period. The second row of boxes record events that occurred in the 30-day period after discharge. There were 159 index admissions, after which the patient did not come into the system at all within 30 days (some of them had clinic visits $>30$ days after discharge, and some of them failed to follow up at all). NSGY = neurosurgery; OR = operating room; plan/unplan = planned/unplanned; VP = ventriculoperitoneal.

status to be statistically significant risk factors (OR 1.39, $\mathrm{p}=0.008$; and OR 1.31, p =0.037, respectively). Eightyseven of the 375 return to ED visits were made by patients with both of these risk factors.

Using shunt reoperation as a dependent variable (n $=157)$, multivariate logistic regression found that afterhour surgery was a statistically significant risk factor ( $\mathrm{p}$ $=0.044$, OR 1.45) whereas subdural and cystoperitoneal shunts were associated with a protective effect from reoperation $(\mathrm{p}=0.035$, OR 0.28$)$.

\section{Discussion}

\section{Rationale of the Study}

This is a descriptive study of clinical events occurring within 30 days of discharge after shunt surgery. It determines the proportion of readmissions that are relevant to the neurosurgical service, the overestimation of which would exaggerate the cost of readmissions. ${ }^{2,14}$ Also, it helps to determine other opportunities to improve patient care. Although lowering shunt revision rates is undoubtedly desirable, it can be questioned whether shunt revision rates can be further decreased without significant technological advances. In an era when hospitals and physicians are under increasing pressure to propose strat- egies to improve patient care, physicians should be at the forefront of these efforts to ensure the feasibility of such quality improvement initiatives.

\section{Comparing Results of This Study to Others}

In this study, the all-cause readmission rate within 30 days of index shunt surgery was $16.5 \%$. Rates of shunt reoperations $(\mathrm{n}=157)$ and all-neurosurgery reoperations $(n=165)$ were $8.9 \%$ and $9.4 \%$, respectively. Readmissions that were deemed to be related to the index surgery (neurosurgical shunt procedures and nonoperative neurosurgical admissions) accounted for $74.5 \%$ of all readmissions.

The National Association of Children's Hospitals and Related Institutions (NACHRI) data set consisted of data contributed to by 72 acute-care children's hospitals from 34 states. An important feature of the NACHRI database is that it uses the "All Patient, Severity-Adjusted Diagnosis-Related Group" (APS-DRG) to classify patients into disease groups. In a NACHRI study, ${ }^{3}$ in the 30 -day period after 3475 ventricular shunt procedures, there were 675 all-cause readmissions (18.1\%) and 262 ventricular shunt procedures (reoperation rate 262 of 3475 [7.5\%]). Interestingly, there were also 89 craniotomies in this 30day period. The high number of craniotomies within 30 days of shunt surgery arouses suspicion that some of the 


\section{Return to system after shunt surgery}

TABLE 2: Univariate analysis of clinical and socioeconomic factors associated with shunt revision and return to ED in patients with hydrocephalus*

\begin{tabular}{|c|c|c|c|c|}
\hline \multirow[b]{3}{*}{ Independent Variable } & \multicolumn{4}{|c|}{ Dependent Variable } \\
\hline & \multicolumn{2}{|c|}{ Shunt Revision Op } & \multicolumn{2}{|c|}{ Return to ED } \\
\hline & OR $(95 \% \mathrm{Cl})$ & $\mathrm{p}$ Value & $\mathrm{OR}(95 \% \mathrm{Cl})$ & $p$ Value \\
\hline ED admission & $1.89(1.31-2.71)$ & 0.001 & $1.32(1.04-1.68)$ & 0.023 \\
\hline PICU admission & $1.07(0.71-1.63)$ & 0.738 & $1.08(0.81-1.44)$ & 0.607 \\
\hline NICU admission & $0.85(0.45-1.61)$ & 0.614 & $1.03(0.68-1.56)$ & 0.906 \\
\hline age & $1.02(0.99-1.04)$ & 0.208 & $1.00(0.98-1.02)$ & 0.760 \\
\hline primary language other than English & $1.07(0.58-1.99)$ & 0.825 & $1.56(1.04-2.33)$ & 0.030 \\
\hline Caucasian & $1.34(0.97-1.93)$ & 0.072 & $1.13(0.89-1.43)$ & 0.330 \\
\hline Atlanta zip codes & $1.17(0.83-1.65)$ & 0.372 & $1.40(1.10-1.78)$ & 0.006 \\
\hline income levels in $\$ 10,000$ s & $0.94(0.84-1.05)$ & 0.278 & $1.04(0.96-1.12)$ & 0.326 \\
\hline Medicaid payee & $0.91(0.65-1.28)$ & 0.597 & $1.34(1.05-1.71)$ & 0.018 \\
\hline after-hour op & $1.79(1.26-2.53)$ & 0.001 & $1.38(1.07-1.77)$ & 0.013 \\
\hline op length $>70$ mins & $0.94(0.54-1.65)$ & 0.840 & $0.76(0.51-1.15)$ & 0.199 \\
\hline VAS op & $1.35(0.72-2.52)$ & 0.345 & $1.28(0.91-2.02)$ & 0.289 \\
\hline externalized shunt/EVD & $0.66(0.29-1.53)$ & 0.335 & $0.69(0.39-1.20)$ & 0.190 \\
\hline subdural/cyst shunt & $0.23(0.07-0.74)$ & 0.014 & $0.74(0.46-1.20)$ & 0.229 \\
\hline spina bifida & $0.39(0.16-0.96)$ & 0.041 & $1.15(0.75-1.75)$ & 0.522 \\
\hline new shunt & $0.60(0.39-0.94)$ & 0.025 & $0.84(0.64-1.12)$ & 0.237 \\
\hline LOS $\geq 3$ days & $0.87(0.61-1.23)$ & 0.438 & $1.29(1.02-1.64)$ & 0.036 \\
\hline
\end{tabular}

* Statistically significant $p$ values are in boldface. PICU = pediatric ICU.

shunt revisions may have been coded as craniotomies. When this category is included, the all-neurosurgery reoperation rate was $10.1 \%([89+262] / 3475)$. Last, when an index admission was categorized under ventricular shunt procedure, $72.4 \%$ of readmissions shared the same index diagnosis and were presumably related to readmission. Overall, despite differences in data processing and definitions, NACHRI and our study yielded similar results.

In the most recently published report from the Pediatric National Surgical Quality Improvement Program (Pediatric NSQIP) ${ }^{13}$ the morbidity rate after neurosurgical procedures, including readmissions and reoperations, was $18 \%$. Surgical site infection was reported to be $6.3 \%$ for neurosurgical procedures, in contrast to $3 \%$ for all other specialties. Approximately half of the neurosurgery procedures were shunt related. The Pediatric NSQIP data again highlight the fact that pediatric neurosurgical pro-

TABLE 3: Multivariate analysis of clinical factors associated with shunt revision and return to ED in patients with hydrocephalus*

\begin{tabular}{lcc}
\hline & \multicolumn{2}{c}{ Dependent Variable: Shunt Revision Op } \\
\cline { 2 - 3 } Independent Variable & $\mathrm{OR}(95 \% \mathrm{Cl})$ & $\mathrm{p}$ Value \\
\hline ED admission & $1.44(0.97-2.11)$ & 0.069 \\
after-hour op & $1.45(1.01-2.09)$ & $\mathbf{0 . 0 4 4}$ \\
subdural/cyst shunt & $0.28(0.09-0.91)$ & 0.035 \\
myelomeningocele & $0.40(0.16-1.06)$ & 0.052 \\
new shunt & $0.76(0.48-1.21)$ & 0.242 \\
\hline
\end{tabular}

* Statistically significant $p$ values are in boldface. cedures, especially those related to ventricular shunts, are under increasing scrutiny.

\section{At-Risk Patient Groups Versus Preventability}

Although there are continuous efforts from the neurosurgical community to decrease shunt malfunction and infection rates, technical failure alone cannot account for all early returns to the system by patients who have undergone shunt placement. Our own data demonstrated that less than half of all shunt patients returning to the ED within 30 days needed subsequent operative interventions. Reductions in inefficient and costly early returns to the hospital will require more than just technical improvements in the care of patients who receive shunts.

TABLE 4: Multivariate analysis of socioeconomic factors associated with shunt revision and return to ED in patients with hydrocephalus*

\begin{tabular}{lcc}
\hline \multirow{2}{*}{\multicolumn{1}{c}{ Independent Variable }} & \multicolumn{2}{c}{$\begin{array}{c}\text { Dependent Variable: } \\
\text { Return to ED }\end{array}$} \\
\cline { 2 - 3 } & OR $(95 \% \mathrm{Cl})$ & $\mathrm{p}$ Value \\
\hline ED admission & $1.17(0.91-1.52)$ & 0.222 \\
primary language other than English & $1.39(0.92-2.10)$ & 0.120 \\
Atlanta zip codes & $1.39(1.09-1.77)$ & $\mathbf{0 . 0 0 8}$ \\
Medicaid payee & $1.31(1.02-1.69)$ & $\mathbf{0 . 0 3 7}$ \\
after-hour op & $1.30(0.94-1.70)$ & 0.560 \\
LOS $\geq 3$ days & $1.28(0.96-1.64)$ & 0.065 \\
\hline
\end{tabular}

* Statistically significant $p$ values are in boldface. 


\section{J. J. Chern et al.}

Not surprisingly, when return to the ED was examined as a clinical event independent of subsequent surgical intervention, different sets of associative risk factors were identified than those noted with shunt failure. Proximity to the hospital and public assistance payee status correlated with a higher likelihood of return visits to the ED. Whereas proximity may represent a bias of the study related to data collection, public assistance payee status represents a surrogate of socioeconomic standing that has been verified in other studies. Education and income have been shown to correlate strongly with ED usage, regardless of the actual cost or availability of emergency services within the region. ${ }^{15,17}$ These variables represent socioeconomic modifiers that future efforts to reduce rebound to the ED in patients treated with shunts will have to consider, and they have already been identified as relevant for other disease entities as well. ${ }^{11,12}$

In certain institutions, patient literacy programs and early postoperative appointments have been implemented, with some success, to bridge the gap in education and social support suffered by these patients. Where they are used, these efforts have produced significant reductions in postoperative early returns to the ED. ${ }^{4,8,9}$ Similar programs might be used for shunt-treated patients of comparable socioeconomic levels to improve their transition from hospitalization to home. However neurosurgeons and health care administrators choose to address this risk factor, our data indicate that ED use among shunt patients is likely to have a large socioeconomic component besides medical necessity, and unilateral efforts from physicians alone are not likely to have a significant impact.

With regard to modifiable variables that do lie almost entirely within the surgeon's control, numerous studies have attempted to identify areas for technical improvement in shunt surgeries. Regarding the mechanical function of shunts, young patient age and the cause of hydrocephalus were most consistently found to be significant prognostic factors, but they are not modifiable. Other clinical factors, such as the position of the ventricular catheter, the shunt valve design, surgeon case volume, and use of intraoperative navigation aids, were inconsistently found to be associated with shunt malfunctions. In this study, we documented the 30-day shunt revision rate to be $8.9 \%$. This is consistent with past studies that suggested shunt malfunction generally decreases over time.,6,16,18

We found that after-hour surgery was a significant risk factor for reoperation within 30 days. This was significant even after adjusting for the presence of myelomeningocele and different types of shunts. After-hour surgery is likely to be associated with poor clinical status at presentation, but it could also be associated with a lower quality of care rendered in the operating room. Unfortunately, this study was not designed to differentiate these important variables. Regimentation among operative teams has certainly been shown to reduce the rate of postoperative complications, and biasing shunt surgeries toward periods when operative teams with the greatest familiarity with shunt surgery are available may lead to fewer early technical failures. Future studies will be needed to further establish this hypothesis.

Still, this paper provides the groundwork for future research into preventing early returns of shunt-treated patients to the health care system. We must stress that this is an associative study meant to identify potential modifiable variables related to early patient return. It was not designed to prove preventability. These data offer insights into systems flow aspects of neurosurgical shunt care (patient health care education and surgical timing) that relate to unintentional and costly returns to the system for patients treated with shunts.

\section{Limitations of the Study}

From the standpoint of studying shunt malfunction, additional clinical factors such as location of the shunt catheter, cause of hydrocephalus, number of previous revisions, use of intraoperative aids, types of valves, and others are obviously desirable. One may also question the quality of coding data in the administrative database. In this study, we avoided using more questionable elements, such as admission and discharge diagnosis, that were recorded in the International Classification of Diseases (ICD) format, but assumed others to be accurate. A prospective multicenter study with precise coding definitions will enable a more balanced view of the interactions between socioeconomic and clinical factors. Another limitation related to a single-institution study is the small number of treating physicians. There might be other practice patterns that are not used in this locality.

\section{Conclusions}

Of the readmissions within 30 days of shunt surgery, $74.5 \%$ were related to the index shunt surgery. The shunt revision rate in the 30 -day period was $8.9 \%$. When adjudicating whether these readmissions are preventable, one has to take basal shunt malfunction rates and the significance of other clinical events into consideration. Further study is needed to identify modifiable risk factors that may eventually improve patient care.

\section{Disclosure}

The authors report no conflict of interest concerning the materials or methods used in this study or the findings specified in this paper.

Author contributions to the study and manuscript preparation include the following. Conception and design: Chern, Tejedor-Sojo. Acquisition of data: Bookland, Riley. Analysis and interpretation of data: Chern. Drafting the article: Chern, Bookland. Critically revising the article: all authors. Reviewed submitted version of manuscript: all authors. Approved the final version of the manuscript on behalf of all authors: Chern. Statistical analysis: Chern, Shoja.

\section{References}

1. Al-tamimi Y, Sinha P, Mathew R, Crimmins D, Thorne J, Hayward R, et al: National audit of ventriculoperitoneal shunt survival in children in the United Kingdom: 30-day failure rate. J Neurosurg 119:A561, 2013 (Abstract)

2. Amin BY, Tu TH, Schairer WW, Na L, Takemoto S, Berven S, et al: Pitfalls of calculating hospital readmission rates based on nonvalidated administrative data sets. Clinical article. J Neurosurg Spine 18:134-138, 2013

3. Berry JG, Toomey SL, Zaslavsky AM, Jha AK, Nakamura 


\section{Return to system after shunt surgery}

MM, Klein DJ, et al: Pediatric readmission prevalence and variability across hospitals. JAMA 309:372-380, 2013 (Erratum in JAMA 309:986, 2013)

4. Coleman EA, Parry C, Chalmers S, Min SJ: The care transitions intervention: results of a randomized controlled trial. Arch Intern Med 166:1822-1828, 2006

5. Feudtner C, Pati S, Goodman DM, Kahn MG, Sharma V, Hutto JH, et al: State-level child health system performance and the likelihood of readmission to children's hospitals. J Pediatr 157:98.e1-102.e1, 2010

6. Garton HJ, Kestle JR, Drake JM: Predicting shunt failure on the basis of clinical symptoms and signs in children. J Neurosurg 94:202-210, 2001

7. Hain PD, Gay JC, Berutti TW, Whitney GM, Wang W, Saville BR: Preventability of early readmissions at a children's hospital. Pediatrics 131:e171-e181, 2013

8. Herman A, Jackson P: Empowering low-income parents with skills to reduce excess pediatric emergency room and clinic visits through a tailored low literacy training intervention. J Health Commun 15:895-910, 2010

9. Jack BW, Chetty VK, Anthony D, Greenwald JL, Sanchez GM, Johnson AE, et al: A reengineered hospital discharge program to decrease rehospitalization: a randomized trial. Ann Intern Med 150:178-187, 2009

10. Joynt KE, Jha AK: Thirty-day readmissions-truth and consequences. N Engl J Med 366:1366-1369, 2012

11. Lippmann SJ, Yeatts KB, Waller AE, Hassmiller Lich K, Travers D, Weinberger M, et al: Hospitalizations and return visits after chronic obstructive pulmonary disease ED visits. Am J Emerg Med 31:1393-1396, 2013

12. Newton AS, Ali S, Johnson DW, Haines C, Rosychuk RJ, Keaschuk RA, et al: Who comes back? Characteristics and predictors of return to emergency department services for pediatric mental health care. Acad Emerg Med 17:177-186, 2010
13. Raval MV, Dillon PW, Bruny JL, Ko CY, Hall BL, Moss RL, et al: Pediatric American College of Surgeons National Surgical Quality Improvement Program: feasibility of a novel, prospective assessment of surgical outcomes. J Pediatr Surg 46:115-121, 2011

14. Shah MN, Stoev IT, Sanford DE, Gao F, Santiago P, Jaques $\mathrm{DP}$, et al: Are readmission rates on a neurosurgical service indicators of quality of care? Clinical article. J Neurosurg 119:1043-1049, 2013

15. Shah SM, Cook DG: Socio-economic determinants of casualty and NHS Direct use. J Public Health (Oxf) 30:75-81, 2008

16. Shah SS, Hall M, Slonim AD, Hornig GW, Berry JG, Sharma $\mathrm{V}$ : A multicenter study of factors influencing cerebrospinal fluid shunt survival in infants and children. Neurosurgery 62:1095-1103, 2008

17. Tozer AP, Belanger P, Moore K, Caudle J: Socioeconomic status of emergency department users in Ontario, 2003 to 2009. CJEM 15:1-7, 2013

18. Tuli S, Drake J, Lawless J, Wigg M, Lamberti-Pasculli M: Risk factors for repeated cerebrospinal shunt failures in pediatric patients with hydrocephalus. J Neurosurg 92:31-38, 2000

Manuscript submitted September 24, 2013.

Accepted February 4, 2014.

Please include this information when citing this paper: published online March 14, 2014; DOI: 10.3171/2014.2.PEDS13493.

Address correspondence to: Joshua J. Chern, M.D., Ph.D., Children's Healthcare of Atlanta, 5455 Meridian Mark Rd. NE, Ste. 540, Atlanta, GA 30342. email: Joshua.Chern@choa.org. 